\title{
Meshless Eigenvalue Analysis for Resonant Structures Based on the Radial Point Interpolation Method
}

\author{
Thomas Kaufmann" ${ }^{\# 1}$ Christophe Fumeaux*2, Christian Engström \#, Ruediger Vahldieck \# \\ \# Laboratory for Electromagnetic Fields and Microwave Electronics (IFH), \\ ETH Zurich, Gloriastrasse 35, CH-8092 Zurich, Switzerland \\ ${ }^{1}$ thomas.kaufmanneifh.ee.ethz.ch \\ ${ }^{*}$ School of Electrical \& Electronic Engineering, The University of Adelaide, \\ Adelaide, South Australia 5005, Australia \\ ${ }^{2}$ cfumeaux@eleceng.adelaide.edu.au
}

\begin{abstract}
Meshless methods are a promising field of numerical methods recently introduced to computational electromagnetics. The potential of conformal and multi-scale modeling and the possibility of dynamic grid refinements are very attractive features that appear more naturally in meshless methods than in classical methods. The Radial Point Interpolation Method (RPIM) uses radial basis functions for the approximation of spatial derivatives. In this publication an eigenvalue solver is introduced for RPIM in electromagnetics. Eigenmodes are calculated on the example of a cylindrical resonant cavity. It is demonstrated that the computed resonance frequencies converge to the analytical values for increasingly fine spatial discretization. The computation of eigenmodes is an important tool to support research on a timedomain implementation of RPIM. It allows a characterization of the method's accuracy and to investigate stability issues caused by the possible occurrence of non-physical solutions.
\end{abstract}

Index Terms-Meshless Methods, Eigenvalues and eigenfunctions, Radial Basis Functions, Radial Point Interpolation Method.

\section{INTRODUCTION}

Meshless Methods for Computational Electromagnetics (CEM) gained attention recently as a new versatile technique for solving the Maxwell equations [1]. The underlying principle avoids an explicit mesh structure for the numerical solution of the differential equations. Instead a set of node locations is selected depending on the physical model at hand. The flexibility in the node distribution allows for conformal and multi-scale modeling. The class of meshless methods is established in other fields of computational physics, e.g. in fluid dynamics or computational mechanics [2]. In CEM in the time domain, recent approaches include Smooth Particle Hydrodynamics for Electromagnetics (SPEM) [3], [4] and the Radial Point Interpolation Time-Domain (RPITD) Method [5], [6]. All these approaches have in common that a node-based interpolation scheme describes the connectivity between the nodes of the computational domain.

The method presented in this publication is a domain discretization collocation method with interpolations based on radial basis functions. It has been introduced as the Kansa RBF method [7], or the Radial Point Interpolation Method (RPIM) [2]. The primary objective of the work of the authors focuses on a time-domain implementation of the RPIM method for electromagnetics. As a matter of fact, the advantages over classical methods, such as geometrical versatility and

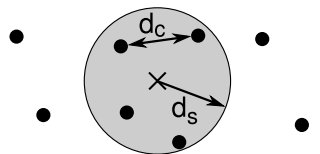

Fig. 1. Support domain of a given node $\times$, showing the area of influence that only includes nodes within radius $d_{s}$. The average node distance $d_{c}$ is used for normalization of radial basis functions.

the dynamic grid adaptation will be best expressed in timedomain applications to simulate transient and possibly multiphysics effects. In this scope an eigenvalue analysis presents a crucial tool to examine accuracy and investigate the presence of spurious modes which eventually can lead to instabilities in the time iteration. Furthermore, eigenmode solvers are an essential tool to characterize arbitrary shaped ports.

The following two sections firstly introduce the interpolation method used in RPIM and secondly their implementation for an eigenvalue problem. Subsequently the approach is analyzed in terms of accuracy and convergence in the example of a twodimensional cylindrical resonator.

\section{RADIAL POINT INTERPOLATION}

The interpolation scheme used in RPIM is based on local radial basis functions (RBFs). This type of basis functions provides excellent interpolation accuracy and an approximation of the spatial derivations can be obtained relatively simply. Only field values in the vicinity of each node inside a local support domain are considered for interpolation, leading to fast local calculations. Fig. 1 depicts the local support domain with radius $d_{s}$. The interpolation of the spatial derivatives are used to obtain the curl operators of the Maxwell equations in differential form.

The used method is thoroughly described in [2] and therefore only briefly summarized here. A field component $u(\mathbf{x})$ at position $\mathbf{x}$ is interpolated as

$\langle u(\mathbf{x})\rangle=\sum_{n=1}^{N} a_{n} r_{n}(\mathbf{x})+\sum_{m=1}^{M} b_{m} p_{m}(\mathbf{x})=\mathbf{r}(\mathbf{x})^{T} \mathbf{a}+\mathbf{p}^{T}(\mathbf{x}) \mathbf{b}$.

The radial basis functions

$$
r_{n}(\mathbf{x})=\exp \left(-\alpha_{c} \frac{\left|\mathbf{x}_{n}-\mathbf{x}\right|^{2}}{d_{c}^{2}}\right)
$$


are of Gaussian type with shape parameter $\alpha_{c}$. The normalization factor $d_{c}$ is the average node distance within the support domain and can be approximated with knowledge of the support domain area $A_{s}$ and the number of neighbors $\left(n_{A_{s}}\right)$

$$
d_{c}=\frac{\sqrt{A_{s}}}{\sqrt{n_{A_{s}}}-1} .
$$

The monomial basis functions $p_{m}(\mathbf{x})$ in (1) are of the following types:

$$
\begin{array}{ll}
\text { zero order: } \mathbf{p}^{T}=[] & (M=0) \\
\text { first order: } \mathbf{p}^{T}=\left[1, x_{m}-x, y_{m}-y\right] & (M=3) .
\end{array}
$$

The interpolation parameters $\mathbf{a}, \mathbf{b}$ are calculated as follows. The vector form of (1) is used to express the interpolated field values for all considered neighbors $\mathbf{U}_{s}=\left(u_{1}, u_{2}, \ldots, u_{n}\right)^{T}$ in the matrix form

$$
\left(\begin{array}{c}
\mathbf{U}_{s} \\
\mathbf{0}
\end{array}\right)=\left(\begin{array}{cc}
\mathbf{R}_{0} & \mathbf{P}_{m} \\
\mathbf{P}_{m}^{T} & \mathbf{0}
\end{array}\right)\left(\begin{array}{l}
\mathbf{a} \\
\mathbf{b}
\end{array}\right)=\mathbf{G}\left(\begin{array}{l}
\mathbf{a} \\
\mathbf{b}
\end{array}\right)
$$

including the constraint condition $\mathbf{P}_{m} \mathbf{a}=\mathbf{0}$. Thus, the interpolation parameters can be calculated by inverting the local matrix $\mathbf{G}$

$$
\left(\begin{array}{l}
\mathbf{a} \\
\mathbf{b}
\end{array}\right)=\mathbf{G}^{-1}\left(\begin{array}{c}
\mathbf{U}_{s} \\
\mathbf{0}
\end{array}\right)
$$

and shape function $\boldsymbol{\Psi}$ is subsequently obtained as

$$
\begin{aligned}
&\langle u(\mathbf{x})\rangle=\left(\mathbf{r}^{T}(\mathbf{x}), \mathbf{p}^{T}(\mathbf{x})\right)\left(\begin{array}{l}
\mathbf{a} \\
\mathbf{b}
\end{array}\right)= \\
& \quad\left[\mathbf{r}^{T}(\mathbf{x}), \mathbf{p}^{T}(\mathbf{x})\right] \mathbf{G}^{-1}\left(\begin{array}{c}
\mathbf{U}_{s} \\
\mathbf{0}
\end{array}\right)=\mathbf{\Psi}(\mathbf{x}) \mathbf{U}_{s} .
\end{aligned}
$$

Similarly, the approximation of the spatial derivations along $\kappa=x, y$ can be expressed as

$$
\left\langle\partial_{\kappa} u(\mathbf{x})\right\rangle=\left[\partial_{\kappa} \mathbf{r}^{T}(\mathbf{x}), \partial_{\kappa} \mathbf{p}^{T}(\mathbf{x})\right] \mathbf{G}^{-1}\left(\begin{array}{c}
\mathbf{U}_{s} \\
\mathbf{0}
\end{array}\right)=\partial_{\kappa} \mathbf{\Psi}(\mathbf{x}) \mathbf{U}_{s} .
$$

The shape parameter $\alpha_{c}$ in (2) influences the condition number of matrix $\mathbf{G}$ and the interpolation accuracy. A suitable value is a balance between high accuracy and bad matrix condition number.

In the practical implementation, a staggered node distribution is well adapted to store $\mathrm{E}$ and $\mathrm{H}$ field components and the $\mathrm{E}$ and $\mathrm{H}$ shape functions have to be calculated separately. The spatial derivatives of the shape functions $\partial_{\kappa} \boldsymbol{\Psi}$ can be expressed as follows: For the E node $i$ the value of the shape function at each neighbor $\mathcal{N}_{e_{i}}$ within the support domain at position $\mathbf{x}\left(\mathcal{N}_{e_{i}}\right)$ is expressed as $\partial_{\kappa} \Psi e_{\mathcal{N}_{e_{i}}}^{i}$. Vice versa, the shape function for $\mathrm{H}$ node $j$ at neighbor positions $\mathbf{x}\left(\mathcal{N}_{h_{j}}\right)$ is expressed as $\partial_{\kappa} \Psi h_{\mathcal{N}_{h_{j}}}^{j}$.

\section{EIGENVALUE SOLVER}

The discretized conservative and source-free Maxwell equations in the frequency domain can be written as

$$
j \omega \mathbf{M}\left(\begin{array}{l}
\mathbf{E} \\
\mathbf{H}
\end{array}\right)=\mathcal{L}\left(\begin{array}{l}
\mathbf{E} \\
\mathbf{H}
\end{array}\right)
$$

with a diagonal material mass matrix $\mathbf{M}=\operatorname{diag}(\varepsilon, \mu)$ and the stiffness matrix $\mathcal{L}=\left(\begin{array}{cc}\mathbf{0} & \nabla \times \\ -\nabla \times & \mathbf{0}\end{array}\right)$ containing the curl operators. The vectors $\mathbf{E}$ and $\mathbf{H}$ contain the field components for all nodes of the electric and magnetic field. The eigenvalues correspond to the resonance frequencies $\omega=-j \lambda$ of the resonator. The eigenvectors represent the field distribution of each mode.

In a two-dimensional TM case with the $z$-independent field components $H_{x}, H_{y}$ and $E_{z}$, the problem reduces to the generalized eigenvalue problem

$$
\lambda \mathbf{M}\left(\begin{array}{c}
\mathbf{E}_{z} \\
\mathbf{H}_{x} \\
\mathbf{H}_{y}
\end{array}\right)=\mathcal{L}\left(\begin{array}{c}
\mathbf{E}_{z} \\
\mathbf{H}_{x} \\
\mathbf{H}_{y}
\end{array}\right) .
$$

The vectors $\mathbf{E}_{z}, \mathbf{H}_{x}$ and $\mathbf{H}_{y}$ are vectors of the length of number of electric and magnetic nodes, respectively. The differential operator matrix in that case contains the spatial derivations in $x$ - and $y$ - direction:

$$
\mathcal{L}=\left(\begin{array}{ccc}
\mathbf{0} & -\partial_{y} \mathbf{L}_{e} & \partial_{x} \mathbf{L}_{e} \\
\partial_{y} \mathbf{L}_{h} & \mathbf{0} & \mathbf{0} \\
-\partial_{x} \mathbf{L}_{h} & \mathbf{0} & \mathbf{0}
\end{array}\right)
$$

The real valued matrices $\partial_{x} \mathbf{L}_{e}, \partial_{y} \mathbf{L}_{e}, \partial_{x} \mathbf{L}_{h}, \partial_{y} \mathbf{L}_{h}$ are obtained by inserting the derivatives of the shape functions (8). $\partial_{\kappa} L_{e}$ and $\partial_{\kappa} L_{h}$ contain entries for $\mathrm{E}$, respectively $\mathrm{H}$ nodes in rows $i, j$ at the column of their neighbors $\mathcal{N}_{e_{i}, h_{j}}$ :

$$
\partial_{\kappa} L_{e}\left(i, k_{i}\right)=\partial_{\kappa} \Psi e_{\mathcal{N}_{e_{i}}}^{i}
$$

and

$$
\partial_{\kappa} L_{h}\left(j, k_{j}\right)=\partial_{\kappa} \Psi h_{\mathcal{N}_{h_{j}}}^{j} .
$$

These matrices are of sparse nature with the number of neighbors $\mathcal{N}_{e, h}$ entries per row.

Boundary conditions for the TM modes are perfect electric conductors (PEC). This Dirichlet boundary is implemented by placing zero-valued $\mathrm{E}$ nodes at the boundary.

\section{NUMERICAL ANALYSIS}

A 2D cavity with circular profile was chosen to demonstrate the validity of our meshless approach. The resonator corresponds to a cylindrical structure with infinite extension in the axial direction. This example was chosen as it utilizes of the conformal modeling abilities of the method and furthermore is a well-known problem with analytical solutions. The TM case is considered with the magnetic field in the cross section and the electric field perpendicular to it. The resonance frequencies of this two dimensional problem are [8]

$$
f_{n m}=\left(\frac{p_{n m}}{r}\right) \frac{c}{2 \pi} .
$$

with the factor $p_{n m}$ being the $m$ th root of the Bessel functions of the first kind $J_{n}(x)=0$ and $r$ the radius of the cavity.

\section{A. Model}

The physical model of the cylindrical cavity with radius $150 \mathrm{~mm}$ is depicted in Fig. 2. The vacuum cavity is enclosed by perfectly electric conducting walls. A set of nodes where 


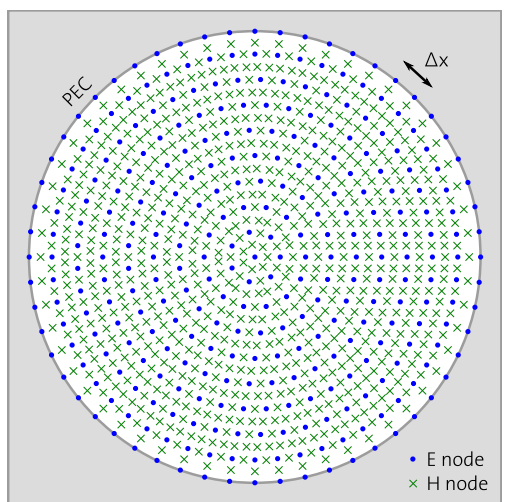

Fig. 2. Depiction of the node arrangement of the cylindrical cavity.

the electric (E) field component is stored is distributed in the cavity volume. The staggered dual magnetic $(\mathrm{H})$ grid is generated by applying a Voronoi tessellation [9] on the E nodes and placing the $\mathrm{H}$ nodes on the edge centers of the resulting polygons. In the case of a structured E node distribution, this would lead to a node distribution comparable to a 2D Yee grid.

\section{B. Simulation}

For each node the support domain first needs to be determined. It is chosen here as a circle with radius of $d_{s}=\alpha_{s} d_{c}$ with $\alpha_{s}=1.1$. This results in an average of 5.7 neighbors per node. A higher number of neighbors increases the accuracy, though at the cost of increased computational complexity. Typical interpolation parameters in (2), (4) are $\alpha_{c}=0.2$ and $M=3$. The accuracy of the boundary is influenced by the choice of the shape parameter and was set accordingly. The values of the shape functions $\partial_{\kappa} \Psi e, h$ can now be calculated from (8). This allows to assemble the stiffness matrix $\mathcal{L}$ (11) and therefore to solve the eigenvalue problem (10). That is achieved in this publication using a full matrix solver [10] in order to guarantee the computation of all eigenvalues and the identification of potential spurious modes. If interested in only a small number of eigenvalues, a sparse iterative solver with a sound initial value might be chosen for a more efficient implementation.

\section{Results}

For a node distance of $\Delta x=15 \mathrm{~mm}$, the distribution of eigenvalues in the complex plane is shown in Fig. 3. The imaginary axis represents the angular frequency $j \omega$, and the analytical solutions (14) are printed in comparison. For low resonance frequencies, i.e. high resolutions in the order of $\lambda / 25$ the eigenvalues match the theoretical value. As frequency grows, the accuracy deteriorates and the eigenvalues are shifted compared to analytical resonance frequencies. Nevertheless their eigenvectors still exhibit symmetrical physical modes.

The electric and magnetic field patterns for a selection of five eigenmodes are presented in Fig. 4 by extracting the field components from the eigenvectors. The field patterns perfectly represent the expected modes. For all modes which allow orthogonal field representations, e.g. $T M_{11}$, degenerate

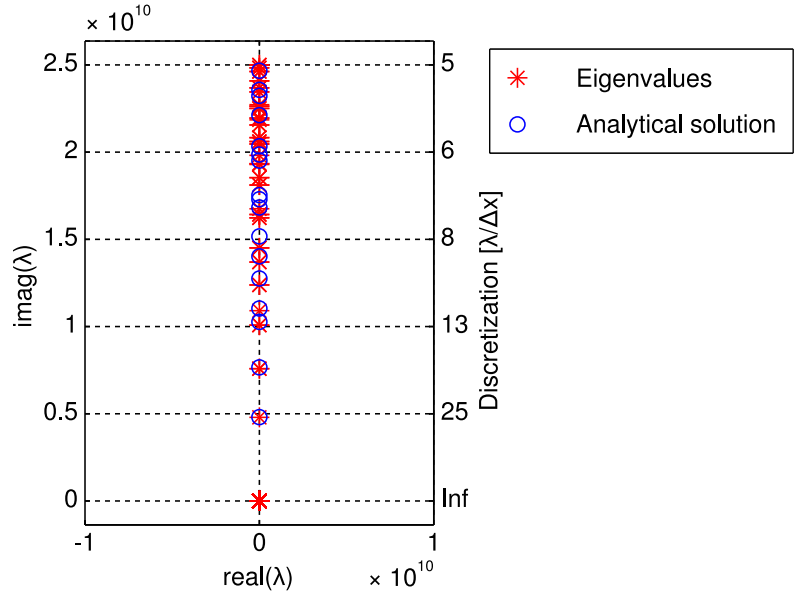

Fig. 3. Eigenmode distribution $\lambda$ for a node distance of $\Delta x=$ $15 \mathrm{~mm}$. The stiffness matrix $\mathcal{L}$ is of size $(1989 \times 1989)$

orthogonal solutions exist, i.e. with a field pattern rotated by $90^{\circ}$.

For the represented eigenmodes, a convergence study of the resonant frequencies has been conducted. The discretization of the model was made gradually finer. Discretizations were chosen between $\Delta x=25 \mathrm{~mm}$ and $\Delta x=7 \mathrm{~mm}$. These node distances correspond to a specific discretization for each mode depending on the resonance frequency. Fig. 5 compares the relative error of the analytical resonant frequencies with the computed eigenvalues. The modes show a good convergence and the errors are much smaller than $0.01 \%$. Error sources are firstly a redshift caused by a phase error in the bulk of the computational domain which becomes significant for resolutions coarser than $\lambda / 10$. Secondly, the circular boundary is approximated by a finite number of nodes, and therefore the effective radius of the resulting polygon is smaller than the corresponding circle, which slightly reduces the resonant frequency.

\section{CONCLUSION}

A meshless eigenvalue solver for the Radial Point Interpolation Method has been implemented and validated on the example of a cylindrical cavity. The field patterns match the physical modes and the resonance frequencies converge with higher resolutions. When solving large problems, using sparse eigensolvers will improve the speed of the algorithm.

The implementation of eigenvalue computation is an essential tool in the development of meshless methods for CEM. In the focus of time-domain simulations, eigenvalues are firstly important to determine the modes in arbitrary shaped ports. Furthermore the eigenmode computations provide an invaluable insight on the properties of the spatial interpolation properties of radial basis functions. The investigation on the possible existence of spurious modes, as well as the understanding of the influence of interpolation parameters on stability and accuracy can be performed more rigorously than in time-domain simulations. 


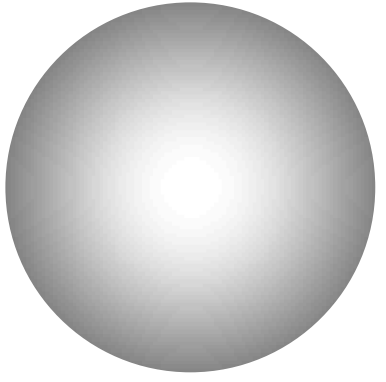

(a) E field for $T M_{01}$

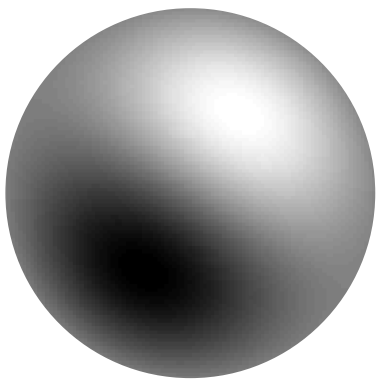

(c) E field for $T M_{11}$

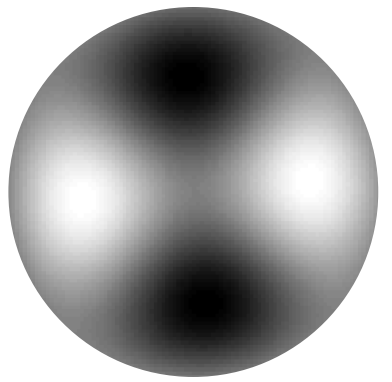

(e) E field for $T M_{21}$

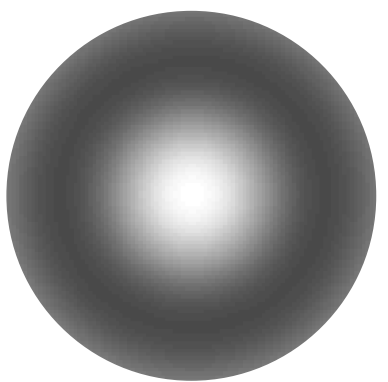

(g) E field for $T M_{02}$

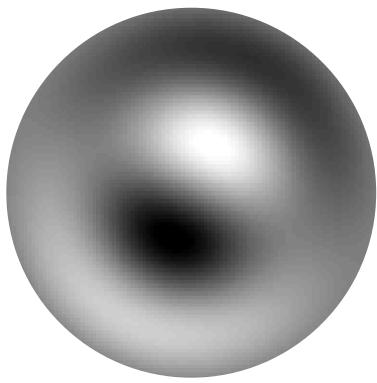

(i) E field for $T M_{12}$

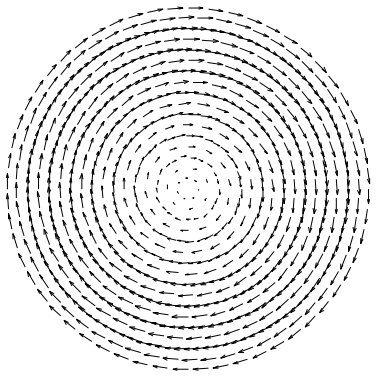

(b) $\mathrm{H}$ field for $T M_{01}$

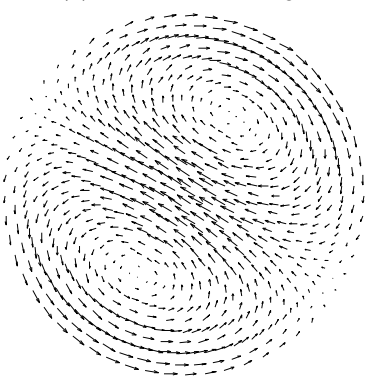

(d) $\mathrm{H}$ field for $T M_{11}$

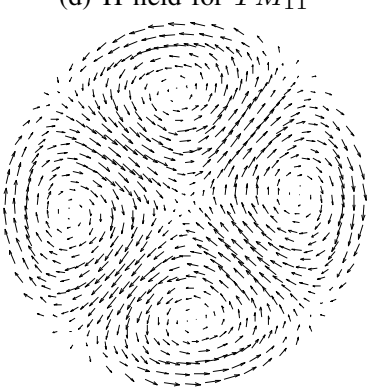

(f) $\mathrm{H}$ field for $T M_{21}$

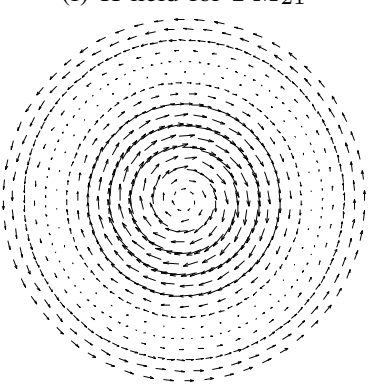

(h) $\mathrm{H}$ field for $T M_{02}$

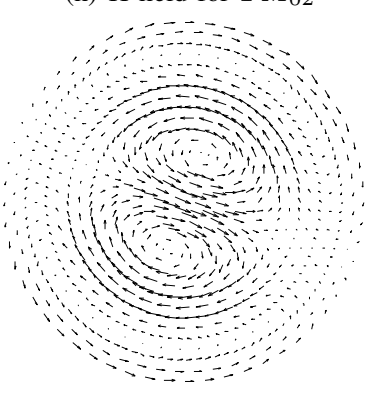

(j) $\mathrm{H}$ field for $T M_{12}$
Fig. 4. A selection of five eigenmodes of the resonant cyclical cavity with radius $r=150 \mathrm{~mm}$.

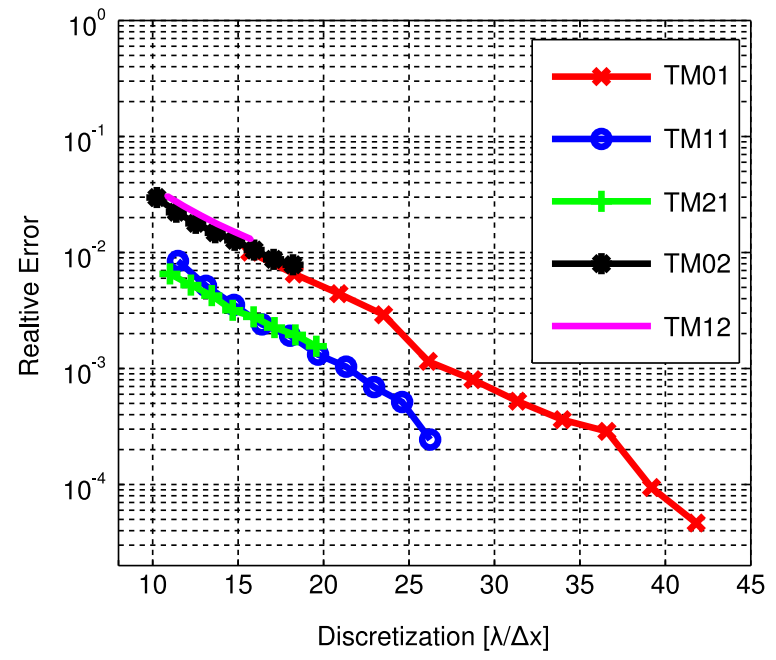

Fig. 5. Convergence study for the error of the eigenvalues of the modes shown in Fig. 4 compared with the analytical resonant frequencies.

\section{ACKNOWLEDGMENT}

This work was performed while Thomas Kaufmann was a visitor at the School of Electrical \& Electric Engineering at the University of Adelaide, Adelaide, South Australia.

\section{REFERENCES}

[1] T. Belytschko,Y. Krongauz, D. Organ, M. Fleming, and P. Krysl, "Meshless method: an overview and recent development," Comput. Methods Appl. Mech. Engrg., Vol. 139, pp. 3-47, 1996.

[2] G.R. Liu and Y.T. Gu, An Introduction to Meshfree Methods and their Programming. Dortrecht, The Netherlands: Springer, 2005.

[3] G. Ala, E. Francomano, A. Tortorici, E. Toscano and F. Viola, "A Smoothed Particle Interpolation Scheme for Transient Electromagnetic Simulation," IEEE Transactions on Magnetics, Vol. 42, No. 4, pp. 647 650, 2006.

[4] K. Krohne, P. Gi-Ho and E.P. Li, "Waveguide Mode Computation through Smoothed Particle Electromagnetics," ACES Conference, Niagara Falls, Canada:ACES, April 2008, pp. 882-887

[5] T. Kaufmann, C. Fumeaux and R. Vahldieck, "The Meshless Radial Point Interpolation Method for Time-Domain Electromagnetics," in IEEE MTT-S Int. Microwave Symposium. Atlanta, GA, USA: IEEE, June 2008, pp. 61-65

[6] Y. Yu and Z. Chen, "An Unconditionally Stable Radial Point Interpolation Method for Efficient Meshless Modeling in Time Domain," ACES Conference, Monterey, CA, USA:ACES, March 2009, pp. 389-394

[7] E.J. Kansa, "Multiquadric scattered data approximation scheme with applications to computational fluid dynamics II," in Computers \& Mathematics with Applications, Vol. 19, No. 8-9, pp. 147-161, 1990.

[8] Robert E. Collin, Foundations for Microwave Engineering, Second Edition, Hoboken, New Jersey: John Wiley \& Sons, 2001.

[9] C. B. Barber, D.P. Dobkin and H. Huhdanpaa, "The quickhull algorithm for convex hulls", ACM Transactions on Mathematical Software (TOMS), Vol. 22, No. 4, pp. 4696-483, 1996

[10] E. Anderson, Z. Bai, C. Bischof, S. Blackford, J. Demmel, J. Dongarra, J. Du Croz, A. Greenbaum, S. Hammarling, A. McKenney, and D. Sorensen, LAPACK User's Guide, Third Edition, SIAM, Philadelphia, 1999. 